\title{
THE EFFECT OF CENTRIFUGAL CONVECTION ON THE STABILITY OF A ROTATING FLUID HEATED FROM BELOW
}

\author{
M. A. TORREST* and J. L. HUDSON \\ Dept. of Chemical Eng. \\ University of Illinois at Urbana-Champaign \\ Urbana, Illinois 61801, U.S.A.
}

\section{$\S 1$. Introduction}

The stability of a fluid layer heated from below and rotated was considered by Chandrasekhar [1] and Chandrasekhar and Elbert [2]. It is assumed that the basic state whose stability is being investigated is solid body rotation with a temperature which is independent of horizontal position and which varies linearly in the vertical. The critical Rayleigh number and the corresponding wave number are determined as functions of the Taylor number. For sufficiently large Prandtl number the onset of convection is stationary $[3,4]$ and the critical Rayleigh number can be predicted by linear theory $[5,6]$. It can be shown by means of a linear stability analysis that for stationary onset at large Taylor numbers the critical Rayleigh number is given by

$$
R \rightarrow P \tau^{\frac{2}{3}} \text { as } \tau \rightarrow \infty
$$

where $R=g \alpha \Delta T d^{3} / v \kappa$, the Taylor number $\tau$ is given by $\tau=4 \omega^{2} d^{4} /$ $\nu^{2}$, and $P$ is a constant; $g$ is the acceleration of gravity, $\alpha$ the coefficient of thermal expansion, $\Delta T$ the imposed temperature difference, $d$ the fluid depth, $v$ the kinematic viscosity, and $\kappa$ the thermal diffusivity. To lowest order the constant $P$ is equal to $3\left(\frac{1}{2} \pi^{2}\right)^{\frac{3}{3}}=$ $=8.6956$ regardless of the boundary conditions on the horizontal surfaces [7]; furthermore, the dimensionless horizontal wave number

* Present address - University of Michigan, Ann Arbor, Michigan 48100 
is $1.305 \tau^{\frac{1}{b}}$ as $\tau \rightarrow \infty$. The mechanism of instability to lowest order is associated with energy conversions entirely within the interior region and the Ekman layers produce higher-order corrections to the limiting critical Rayleigh number [8]; the latter is unaffected by the presence of side walls.

In all the above studies it is assumed that the fluid is in solid body rotation before gravitational instabilities set in. However, solid body rotation cannot occur when a rotating fluid is heated from below regardless of how small the imposed vertical temperature difference may be since motion relative to solid body rotation is produced by the coupling of the vertical density gradient and the centrifugal acceleration. This is analogous to the convection which occurs when a density gradient is imposed normal to the acceleration of gravity in a nonrotating system. Convection in a right circular cylinder which is heated differentially in the vertical and which rotates rapidly about its vertical axis has been analyzed by Barcilon and Pedlosky [9] and by Homsy and Hudson [10, 11]. It is assumed that the flow is laminar and axisymmetric and, except for a part of the work in [11] which is discussed further below, gravitational instabilities are not considered. The dimensionless temperature and velocities depend on five parameters: $\sigma=v / \kappa$ (Prandtl number), $\varepsilon=v / 2 \omega h^{2}$ (Ekman number), $\gamma=a / h$ (aspect ratio), $A=g / \omega^{2} a$ (acceleration ratio or inverse Froude number), and $\beta=\alpha \Delta T / 8$ (thermal Rossby number). $h$ and $a$ are the cylinder half-height and radius respectively. The notation corresponds to that used in [10] and [11]. A grouping of the parameters, viz., $\lambda=\sigma \beta \varepsilon^{-\frac{1}{2}}$, is the ratio of thermal convection to conduction in the interior of the cylinder. Thus for $\lambda$ small, heat is transferred between the horizontal surfaces primarily by conduction; for $\lambda=0$, the fluid is in solid body rotation. When $\lambda$ is large, convection is important. The region of parameter space $\beta \ll 1, \varepsilon \ll 1, A \ll 1$, $\lambda \leqslant O(1)$ was considered in [10] with $1 \leqslant \gamma \leqslant O\left(\varepsilon^{-\frac{1}{8}}\right)$ for conducting side walls and $\gamma=O(1)$ for insulated side walls. In [11], further results are presented for $\beta \ll 1, \varepsilon \ll 1, A \ll(\lambda \gamma)^{-1}, \gamma \gg 1, \lambda \gamma \leqslant O(1)$, i.e., for large aspect ratios but small $\lambda$.

In [11], we made an initial study of gravitational instabilities in a rotating cylinder heated from below including the effect of the centrifugal acceleration. A linear stability analysis was made about a basic state in which convection occurs relative to solid body ro- 
tation and in which the temperature field is a function of both vertical and horizontal positions. Boundary layer methods were used which are valid in the limit of small Ekman number (or equivalently, large Taylor number). Since in this limit the horizontal length scale of the disturbance fields is very small relative to that of the basic state (large wave number), an approximate solution was obtained by solving the stability equations at each radial position taking into account vertical, but not horizontal, variations in the basic state. A "local" critical Rayleigh number was determined at each radial position, calculated as for a radially infinite medium. In the present study, this approximation is not made; i.e., the linearized equations governing the stability of a basic state which varies in both radial and vertical directions are solved by means of the Galerkin method. In order to relate these stability analyses to previous studies, parameters somewhat different from those defined above are used. Thus, as in [8] and [11] we use $\gamma_{0}=a / d=\frac{1}{2} \gamma$ (aspect ratio), $E=\nu / 2 \omega d^{2}=\frac{1}{4} \varepsilon$ (Ekman number), $R=g_{\alpha} A T d^{3} / v \kappa=16 \beta A \gamma \sigma / \varepsilon^{2} \quad$ (Rayleigh number), $\sigma S=v g_{\alpha} \Delta T /$ $4 \kappa \omega^{2} d=R E^{2}$ (stratification parameter).

\section{§ 2. Mathematical analysis}

Consider a right circular cylinder of height $d$ and radius $a$ which rotates around its vertical axis at angular velocity $\omega$. The Taylor number is large. The temperatures of the top and bottom surfaces are $T_{\mathrm{a}}$ and $T_{\mathrm{b}}$ respectively where $T_{\mathrm{b}}>T_{\mathrm{a}}$. When $T_{\mathrm{b}}-T_{\mathrm{a}}$ is less than the value required to produce gravitational instabilities, the resulting centrifugally driven flow field is that discussed in [10] and [11]. It is assumed that the flow is laminar and axisymmetric. The velocity (denoted by $Q^{\prime}$ ) and the temperature (denoted by $\Theta^{\prime}$ ) depend on both vertical and radial position; this situation will be referred to as the basic state. As the temperature difference $T_{\mathrm{b}}-T_{\mathrm{a}}$ is increased, a point is reached at which gravitational instabilities set in. In order to determine this value of $T_{\mathrm{b}}-T_{\mathrm{a}}$, the governing equations are linearized about $Q^{\prime}$ and $\Theta^{\prime}$, and the critical Rayleigh number found. It is assumed that the Prandtl number is greater than about $\sqrt{ } 2$ so that the onset is stationary and adequately predicted by linear theory. Furthermore, in making the Galerkin expansion below it is assumed that the disturbance fields are also axisymmetric. This assumption can be justified by noting that the 
difference between critical Rayleigh numbers for various azimuthal waves numbers decreases as the Taylor number is increased [8] and also by noting Koschmieder's experimental observation that at the onset of instability in a cylindrical container the initial disturbances take the form of axisymmetric roll cells $[12,13,14]$. It is assumed that the fluid is incompressible except in the gravitational and centrifugal terms where it is

$$
\rho=\rho_{0}\left[1-\alpha\left(T-T_{0}\right)\right] .
$$

Linearizing about the basic state $Q^{\prime}\left(r^{\prime}, z^{\prime}\right)$ and $\Theta^{\prime}\left(r^{\prime}, z^{\prime}\right)$ where $r^{\prime}$ and $z^{\prime}$ are dimensional positions, the equations governing the disturbance velocity $q^{\prime}$ and temperature $T^{\prime}$ become

$$
\begin{gathered}
\boldsymbol{\nabla} \cdot \boldsymbol{q}^{\prime}=0, \\
\boldsymbol{Q}^{\prime} \cdot \boldsymbol{\nabla} \boldsymbol{q}^{\prime}+\boldsymbol{q}^{\prime} \cdot \boldsymbol{\nabla} Q^{\prime}+2 \omega\left(\boldsymbol{k} \times \boldsymbol{q}^{\prime}\right)+\boldsymbol{r}^{\prime} \omega^{2} \alpha T^{\prime} \boldsymbol{i}+ \\
-g_{\alpha} T^{\prime} \boldsymbol{k}=-\rho_{0}^{-1} \boldsymbol{\nabla} p^{\prime}+{ }^{2} \nabla^{2} \boldsymbol{q}^{\prime}, \\
\boldsymbol{Q}^{\prime} \cdot \boldsymbol{\nabla} T^{\prime}+\boldsymbol{q}^{\prime} \cdot \boldsymbol{\nabla} \Theta^{\prime}=\kappa \nabla^{2} T^{\prime},
\end{gathered}
$$

where $\boldsymbol{k}$ is the unit vector in the vertical, and $\boldsymbol{i}$ is the unit vector in the radial direction.

Employing a basic state valid as $E \rightarrow 0$, we shall accordingly treat only the asymptotic stability problem. It is shown in [8] that in this case the gravitational instability is associated only with interior energy conversions in which the release of potential energy due to the stratification is balanced by interior horizontal dissipation. Thus, since vertical diffusion and its effect on disturbances is confined to the Ekman layers it is not associated with the instability and we can represent the total basic state (interior with many boundary layer corrections) by its interior term alone.

We introduce dimensionless variables (unprimed)

$$
\begin{aligned}
q^{\prime} & =q\left(\frac{g \alpha \Delta T}{2 \omega}\right) \\
p^{\prime} & =p \rho_{0} g \alpha d \Delta T \\
T^{\prime} & =T(\Delta T) \\
\Theta^{\prime} & =\Theta(\Delta T)
\end{aligned}
$$




$$
\begin{aligned}
& Q^{\prime}=Q\left(\frac{\alpha \Delta T \omega a}{4}\right) \\
& z^{\prime}=z d \\
& r^{\prime}=r d
\end{aligned}
$$

where $\Delta T=T_{\mathrm{b}}-T_{\mathrm{a}}$ and utilize an order of magnitude analysis analogous to that of [11] valid for $\gamma=O(1), \beta<E^{\frac{1}{3}}, \sigma \beta<E^{\frac{1}{3}}$ and $E^{\hat{s}} \ll A \ll(\lambda \gamma)^{-1}$. It is shown in [11] that the inertial and centrifugal terms in (3) and the first term in (4) can be neglected and the dimensionless stability equations reduce to

$$
\begin{gathered}
\boldsymbol{\nabla} \cdot \boldsymbol{q}=0 \\
\boldsymbol{k} \times \boldsymbol{q}=-\nabla p+E \nabla^{2} \boldsymbol{q}+\boldsymbol{k} T \\
\sigma S(\boldsymbol{q} \cdot \boldsymbol{\nabla} \Theta)=E \nabla^{2} T
\end{gathered}
$$

with boundary conditions

$$
\begin{gathered}
\left.\begin{array}{c}
T=0 \\
\boldsymbol{q}=\mathbf{0}
\end{array}\right\} \text { at } z=0 \text { and } 1 \\
\boldsymbol{q}=\mathbf{0}, \text { at } r=r_{0}
\end{gathered}
$$

and either

$$
T=0 \quad \text { at } \quad r=r_{0} \quad \text { (conducting wall) }
$$

or

$$
\frac{\partial T}{\partial r}=0 \quad \text { at } \quad r=r_{0} \quad \text { (insulated wall) }
$$

where $r_{0}=a / d=\gamma / 2, \gamma$ and $z$ are defined in (5), $\sigma, S$, and $E$ are defined in the introduction. Using the fact from (1) that for very small Ekman number

$$
R=P E^{-\frac{4}{3}}
$$

or equivalently

$$
\sigma S=P E^{\frac{2}{3}}
$$

where $P$ is a constant, the set of eqs. (6-8) with boundary conditions (9-12) constitutes an eigenvalue problem for the critical Rayleigh number $R$ or equivalently for the constant $P$ since $P$ and $R$ are related as in (1) or (13). 
The dependent variables in (6-8) are expanded in a series in the small parameter $E^{\frac{b}{b}}$. Introducing the new dimensionless coordinate

$$
x=r E^{-\frac{1}{3}}\left(0 \leqslant r \leqslant r_{0} ; 0 \leqslant x \leqslant r_{0} E^{-\frac{1}{3}}\right)
$$

the zeroth order eigenvalue problem for the interior fields becomes [11]

$$
\begin{gathered}
\frac{\partial w}{\partial z}=-\nabla_{1}^{4} p \\
v=\frac{\partial p}{\partial x}, \quad u=-x^{-1} \frac{\partial p}{\partial \phi} \\
\frac{\partial p}{\partial z}=\nabla_{1}^{2} w+T \\
P\left(u \frac{\partial \Theta}{\partial r}+w \frac{\partial \Theta}{\partial z}\right)=\nabla_{1}^{2} T
\end{gathered}
$$

where

$$
\nabla_{1}^{2}=x^{-1} \frac{\partial}{\partial x} \times \frac{\partial}{\partial x}+x^{-2} \frac{\partial^{2}}{\partial \phi^{2}}
$$

$\phi$ is the aximuthal coordinate, and $u, v, w$ are the lowest order nonzero radial, tangential, and vertical components of the velocity. The boundary conditions at the horizontal surfaces are

$$
\left.\begin{array}{l}
w=0 \\
T=0
\end{array}\right\} \quad \text { at } \quad z=0 \text { and } 1 .
$$

Having assumed an $E^{-\frac{1}{\delta}}$ horizontal variation (dimensionlessly $\left.\partial / \partial r \sim E^{-\frac{1}{3}}\right)$ the interior problem is of sufficiently high order to satisfy the side wall boundary conditions,

$$
u=v=w=0 \quad \text { at } \quad x=r_{0} E^{-\frac{3}{3}}
$$

and either

$$
T=0 \quad \text { at } \quad x=r_{0} E^{-\frac{1}{8}} \quad \text { (conducting wall) }
$$

or

$$
\frac{\partial T}{\partial r}=0 \quad \text { at } \quad x=r_{0} E^{-\frac{1}{3}} \quad \text { (insulated wall) }
$$


Assuming that the disturbances are axisymmetric, equations (16-19) become

$$
\begin{aligned}
& \frac{\partial w}{\partial z}=-x_{0}^{-4} L^{2}(p) \\
& \frac{\partial p}{\partial z}=x_{0}^{-2} L(w)+T \\
& P w \frac{\partial \Theta}{\partial z}=x_{0}^{-2} L(T)
\end{aligned}
$$

where the radial position $r(0 \leqslant r \leqslant 1)$ has been reintroduced,

and

$$
x_{0}=r_{0} E^{-\frac{1}{8}}
$$

$$
L=r^{-1} \frac{\partial}{\partial r} r \frac{\partial}{\partial r}
$$

Eqs. (24-26) are now to be solved for the eigenvalue $P$ in terms of the parameter $x_{0}$. It is noted that $x_{0}=\gamma_{0} E^{-\frac{1}{3}} \gg 1$, since $\gamma_{0}=O(1)$ and $E$ is small. The basic state temperature field $\Theta$ is known from. [10] and [11] and depends primarily on the parameter $\lambda=\sigma \beta \varepsilon^{-\frac{1}{2}}$ and the aspect ratio $r_{0}$. Since $\Theta$ is a function of both vertical and radial positions, an analytical solution is not possible and the Galerkin method is used. Furthermore, since the horizontal length scale of the disturbances is small $\left(O\left(E^{3}\right)\right)$ and since it was shown in [8] that in the case of solid body rotation the side wall does not influence the critical Rayleigh number, a side slip wall boundary condition is used.

and

$$
\begin{aligned}
& p=\sum_{i=A}^{B} \sum_{j=1}^{C} p_{i, j} J_{0}\left(\delta_{i} r\right) \cos (j \pi z) \\
& T=\sum_{i=A}^{B} \sum_{j=1}^{C} T_{i, j} J_{0}\left(\delta_{i} r\right) \sin (j \pi z)
\end{aligned}
$$

$$
w=\sum_{i=A}^{B} \sum_{j=1}^{C} w_{i, j} J_{0}\left(\delta_{i} r\right) \sin (j \pi z)
$$

Recognizing that the radial trial functions describing only a few cells should be relatively unimportant when actually many radial cells exist, the $r$-expansions were initiated with some integer value 
$A$, rather than one. Computation time was thus diminished and satisfactory results were obtained. The value taken for $A$ is dependent on $x_{0}$ and was estimated from the solid body rotation solution [8].

An estimation of $\delta_{m}$ is

$$
\delta_{m}=(m+0.25) \pi=1.305 x_{0} .
$$

The integer $C$ is dependent on the value of $\lambda$ and was taken as 3 or 4 . More will be said about this later. The $\delta_{i}$ in the arguments of (29) are the roots of $J_{1}$, that is

$$
J_{1}\left(\delta_{i}\right)=0, \quad i=1,2, \ldots
$$

Substituting the disturbance expansions into (24-26) noting that

$$
\left(\frac{1}{r} \frac{d}{d r} r \frac{d}{d r}\right) J_{0}\left(\delta_{i} r\right)=-\delta_{i}^{2} J_{0}\left(\delta_{i} r\right)
$$

and requiring the error to be orthogonal to each expansion pair we obtain the Galerkin equations,

$$
\begin{gathered}
(j \pi) w_{i, j}=-\left(\frac{\delta_{i}}{x_{0}}\right)^{4} p_{i, j} \\
-(j \pi) p_{i, j}=-\left(\frac{\delta_{i}}{x_{0}}\right)^{2} w_{i, j}+T_{i, j} \\
P \sum_{k=A}^{B} \sum_{l=1}^{C} w_{k, l} T(i, j, k, l)=-\left(\frac{\delta_{i}}{x_{0}}\right)^{2} T_{i, j}
\end{gathered}
$$

where

$$
\begin{aligned}
& T(i, j, k, l)= \\
& \quad=\frac{4}{J_{0}^{2}\left(\delta_{i}\right)} \int_{0}^{1} \int_{0}^{1} \frac{\partial \Theta}{\partial z} \sin (j \pi z) \sin (l \pi z) J_{0}\left(\delta_{i} r\right) J_{0}\left(\delta_{k} r\right) r \mathrm{~d} r \mathrm{~d} z
\end{aligned}
$$

Eqs. (30-32) can be reduced to the single expression

$$
P \sum_{k=A}^{B} \sum_{l=1}^{C} w_{k, l} T(i, j, k, l)=-\left[\left(\frac{\delta_{i}}{x_{0}}\right)^{4}+(j \pi)^{2}\left(\frac{\delta_{i}}{x_{0}}\right)^{-2}\right] w_{i, j} .
$$

We now let

$$
\Theta=-z-\frac{1}{2} \Phi\left(r, z^{*}\right)
$$


where

$$
z^{*}=2 z-1, \quad-1 \leqslant z^{*} \leqslant 1 .
$$

Since the analytical expression for $\Theta$ (or $\Phi$ ), which is known from [10] or [11], is too complicated to allow the evaluation of the integral (33), the function $\Phi$ is expanded in a Chebyshev polynominal

$$
\Phi\left(r, z^{*}\right)=\sum_{m=0}^{D} \sum_{n=0}^{E} c_{m, n} T_{m}\left(r^{*}\right) T_{n}\left(z^{*}\right)
$$

where $r^{*}$ is the radial coordinate $\left(-1 \leqslant r^{*} \leqslant 1\right)$ related to $r=r^{\prime} \mid a$ by

$$
r^{*}=2 r-1
$$

and the coefficients are given by

with

$$
c_{m, n}=q(m, n) \int_{-1}^{1} \int_{-1}^{1} \frac{\Phi\left(r, z^{*}\right) T_{m}\left(r^{*}\right) T_{m}\left(z^{*}\right) \mathrm{d} r^{*} \mathrm{~d} z^{*}}{\left(1-r^{*}\right)^{\frac{1}{2}}\left(1-z^{*}\right)^{\frac{1}{2}}}
$$

$$
\begin{aligned}
& q(0,0)=1 / \pi^{2} \\
& q(m, 0)=q(0, n)=2 / \pi^{2} \text { for } m, n>0 \\
& q(m, n)=4 / \pi^{2} \text { for } m, n>0 .
\end{aligned}
$$

The double integral (38) is evaluated numerically utilizing the analytical representation for $\Phi$ [8] and a two dimensional ChebyshevGaussian quadrature. The coefficients become

$$
c_{m, n}=\frac{a(m, n)}{N^{2}} \sum_{i=1}^{N} \sum_{j=1}^{N} \Phi\left(\frac{a_{i}+1}{2}, a_{j}\right) T_{m}\left(a_{i}\right) T_{n}\left(a_{j}\right)
$$

where

$$
a_{k}=\cos \left(\frac{(2 k-1) \pi}{2 N}\right)
$$

$N$ is the order of the Gaussian quadrature, and

$$
a(m, n)=\left[\begin{array}{cccc}
1 & 2 & \ldots & 2 \\
2 & 4 & \ldots & 4 \\
\vdots & \cdot & & \cdot \\
\cdots & \ddots & \ldots & . \\
\cdot & . & & . \\
2 & (4) & \ldots & 4
\end{array}\right] \quad \begin{aligned}
& m=0,1, \ldots D \\
& n=0,1, \ldots E
\end{aligned}
$$


A 30th order Gaussian quadrature was employed. The accuracy of the 4-term Chebyshev expansion rapidly decreased with increasing aspect ratio. For although the relative error in the Chebyshev coefficients $c_{m, n}$ is $\pm 5.0 \times 10^{-5}$, the temperature profile error is up to approximately $\pm 1 \%$ for $\gamma=1$, up to $\pm 5 \%$ for $\gamma=5$, and up to $\pm 30 \%$ for $\gamma=10$. The use of larger Chebyshev polynomial expansions was prohibited by the inordinately difficult $R(j, l, n)$ and $S(i, k, m)$ integrals that resulted.

A polynomial representation for $\Theta$ is now available from (35) and (37). This is substituted into (33) to obtain $T(i, j, k, l)$ in the form

where

$$
T(i, j, k, l)=-\delta_{i k} \delta_{j l}+\sum_{m} \sum_{n} c_{m, n} S(i, k, m) R(j, l, n)
$$

$$
S(i, k, m)=\int_{0}^{1} T_{m}\left(r^{*}\right) J_{0}\left(\delta_{i} r\right) J_{0}\left(\delta_{k} r\right) r \mathrm{~d} r
$$

$R(j, l, n)=\int_{0}^{1} T_{n}\left(z^{*}\right)\{(j+l) \sin (j+l) \pi z-(j-l) \sin (j-l) \pi z\} \mathrm{d} z$.

The $R(j, l, n)$ integrals are evaluated analytically by means of repeated integration by parts. In order to do the $S(i, k, m)$ integrals, use is also made of integration formulae and recurrence relations from Luke [15] and Watson [16] and asymptotic representations for Bessel functions of large argument. Details are given in [17].

Since the $T(i, j, k, l)$ are known, (34) is a matrix equation for the eigenvalue $P$ and the eigenvectors $\boldsymbol{w}$. Thirty horizontal and three or four vertical terms were used in the expansions. The resulting unsymmetric matrices were solved for the eigenvalues by Parlett's method [18] and the eigenvectors $\boldsymbol{w}$ were then obtained by inverse iteration [19]. Increasing the number of lateral terms from 30 to 40 resulted in a $\pm 0.001(\sim 0.01 \%)$ deviation in $P$ for $\gamma=O(1)$. The relative difference in $P$ for increasing the number of axial terms from 3 to 4 increased with increasing $\lambda$ from essentially zero at $\lambda=1.0$ to about $3.5 \%$ at $\lambda=10.0$ (for $\gamma=1.0$ ).

\section{§ 3. Basic state}

The flow pattern in a cross section of a right circular cylinder heated from below and rotated about its vertical axis is shown in Fig. 1 [10]. Boundary layers form on all surfaces when the Ekman number $E$ is small (large Taylor number). Temperature profiles computed from (35) and (37) for $\lambda=\sigma \beta \varepsilon^{-\frac{1}{2}}=1.0$ and aspect ratio $\gamma=1.0$ are shown in Fig. 2. As noted in $\S 2$, the stability of the fluid was in- 


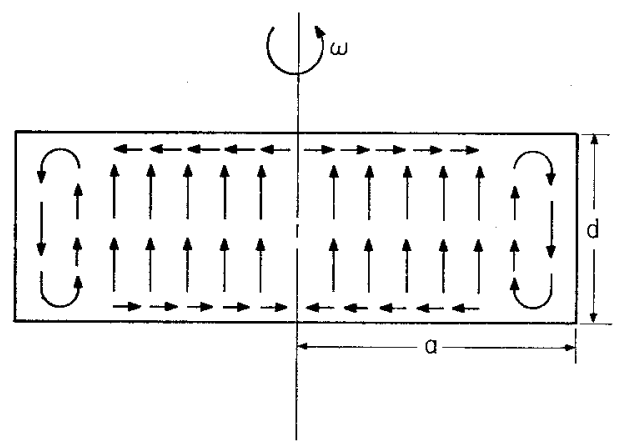

Fig. 1. Basic state radial and axial velocities in a cross section of the cylinder. The Ekman number is small.

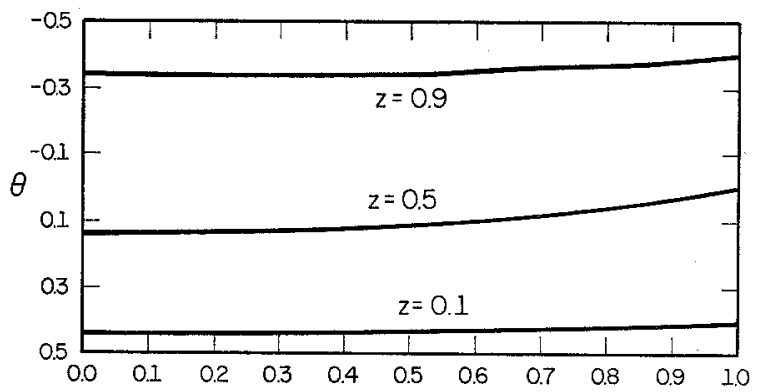

Fig. 2. Temperature profiles for the basic state. Conducting side wall, $\lambda=\sigma \beta \varepsilon^{-\frac{1}{2}}=1.0 ;$ aspect ratio $\gamma=1.0$.

vestigated by linearizing the governing equations about the conditions shown in Figs. 1 and 2 . The parameter $\lambda$ is the ratio of convective to conductive heat transfer in the interior region of the cylinder. Thus for $\lambda=0.0$, the fluid is in solid body rotation and as $\lambda$ is increased, convection becomes important. The temperature depends on both radial and axial positions because of the effect of centrifugally driven circulations. For larger aspect ratio the temperatures expressed by the Chebyshev polynomials oscillate slightly with radial position since only fourth order polynomials were used. As the aspect ratio is increased the radial gradients in $\Theta$ are increasingly confined to the regions near the side wall. The accuracy of the polynomial fit diminishes with increasing aspect ratio, the maximum error being up to $1 \%$ for $\gamma=1$, up to $5 \%$ for $\gamma=5$, and up to $30 \%$ for $\gamma=10$. 


\section{§ 4. Critical Rayleigh numbers}

The above calculations determine the eigenvalue $P$ from which the critical Rayleigh number for large rotational rates is known through (13). In Fig. 3 is shown the results for $P$ for the case $\lambda=\gamma=1.0$ and a conducting side wall. The abscissa is $x_{0}=r_{0} E^{-t}$. Since our results are valid only for small Ekman numbers (large Taylor numbers), we will restrict the discussion to values of $E$ such that $P$ is independent of $x_{0}$, i.e., for this case $x_{0}>3000$.

Some results for $\lambda<1$ and various aspect ratios $\gamma$ are presented in Table 1 for a conducting side wall. $P$ increases with both increasing $\lambda$ and $\gamma$, although in every case except $\lambda=1.0, \gamma=10.0$ the results are within $1 \%$ of the result predicted from a solid body rotation analysis $(P=8.695)$. It is noted that for $\lambda=0$, there is no basic state convection and the solid body rotation results are

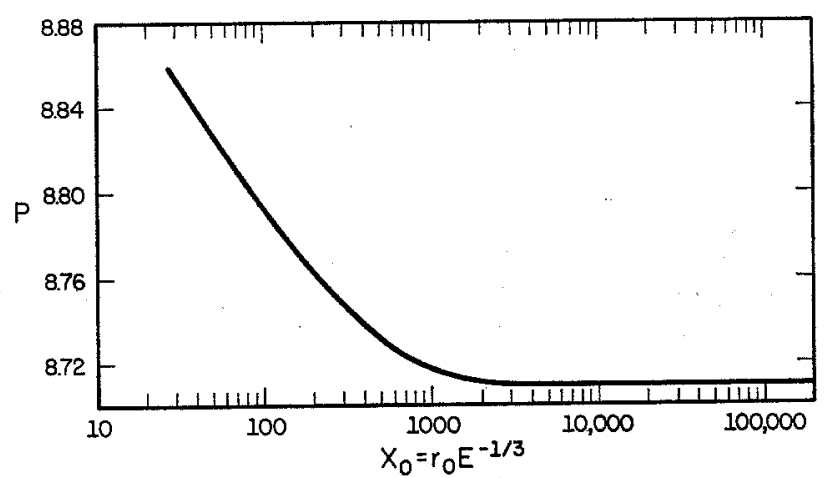

Fig. 3. The constant $P=R E^{\frac{4}{3}}$ verses the parameter $x_{0}=r_{0} E^{-\frac{1}{3}}$. Conducting side wall. $\lambda=\sigma \beta \varepsilon^{-\frac{1}{2}}=1.0$; aspect ratio $\gamma=1.0$.

TABLE I

\begin{tabular}{|c|c|c|c|c|}
\hline \multirow[b]{2}{*}{$\lambda$} & \multicolumn{4}{|c|}{$\gamma=\mathrm{a} / \mathrm{h}$} \\
\hline & 1.0 & 2.0 & 3.0 & 10.0 \\
\hline 0.0 & 8.6959 & 8.6959 & 8.6959 & 8.6959 \\
\hline 0.1 & 8.625 & 8.627 & 8.629 & 8.632 \\
\hline 0.25 & 8.637 & 8.651 & 8.655 & 8.658 \\
\hline 0.50 & 8.670 & 8.707 & 8.717 & 8.758 \\
\hline 1.00 & 8.710 & 8.744 & 8.767 & 8.950 \\
\hline
\end{tabular}

The constant $P$ for a conducting side wall; $x_{0}=2.0 \times 10^{5}$ 
obtained. The eigenvalue calculations are accurate to \pm 0.001 as shown in [17], although this does not include the fact that errors are introduced in approximating the basic state by a polynomial expansion.

The parameter $\lambda$ is the ratio of convective to conductive heat transfer in the interior regions of the basic state. As $\lambda$ increases from 0.1 to 1.0, the value of $P$ also increases. However, the effect is very small. It is noted that the critical Rayleigh number can be less for $\lambda$ small but nonzero than it is for $\lambda=0$. Consider for example an aspect ratio $\gamma=1.0$. For no basic state convection $(\lambda=0.0)$ the value of $P$ is 8.6959 at all aspect ratios. We investigated the range $0 \leqslant \lambda \leqslant 0.1$ to determine the minimum value of $P$ and at what value of $\lambda$ it would occur. For all values of $\lambda$ between $10^{-2}$ and $10^{-6}, P=8.624$ and this was the minimum value. The constant $P$ does not start rising back to its value at $\lambda=0$ until $\lambda=10^{-7}$ at which point $\lambda=8.642$. From the remarks and from Table 1 it is seen that there is little effect of basic state convection on the critical Rayleigh number for $\lambda \leqslant 1.0$. The very slight depression in $P$ for $\lambda \neq 0$ compared to $\lambda=0$ may be caused by the approximation to the basic state; even if the depression is real, it would be difficult to observe experimentally. For $\lambda>1.0$, the effect of basic state convection is to stabilize the layer compared to $\lambda=0$ (solid body rotation basic state). This can be seen in Fig. 4 where $P$ is shown as a function of $\lambda$ for a conducting side wall and various aspect ratios. This can be explained in the following manner.

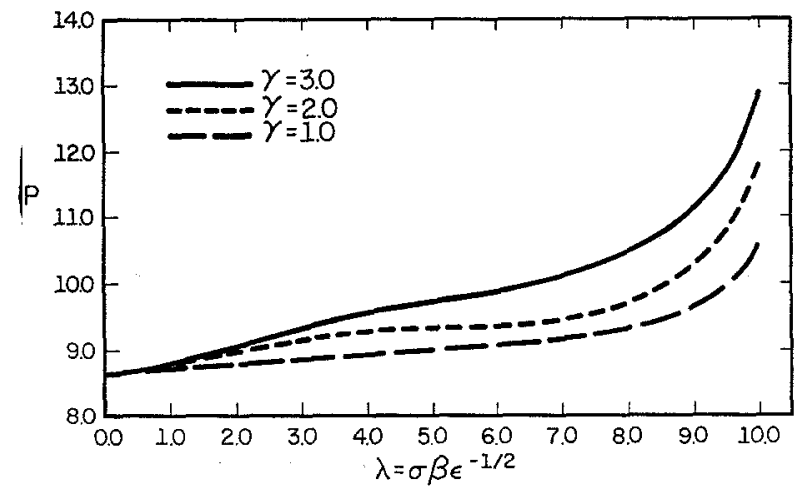

Fig. 4. The constant $P=R E^{\frac{4}{3}}$ verses the parameter $\lambda=\sigma \beta \varepsilon^{-\frac{1}{2}}$ at various aspect ratios $\gamma$. Conducting side wall. 
In the basic state the fluid spirals radially outward near the top horizontal surface, down near the side wall, radially inward near the bottom surface, and up through the interior of the cylinder as shown in Fig. 1. This latter flow moves warm fluid upward and decreases the vertical distance over which a substantial vertical temperature gradient exists. An indication of this is given in Fig. 2. The result of decreasing the characteristic vertical distance is to stabilize the fluid to gravitational instabilities.

TABLE II

\begin{tabular}{ccccc}
\hline \multicolumn{5}{c}{$\gamma=\mathrm{a} / \mathrm{h}$} \\
\cline { 2 - 5 }$\lambda$ & 1.0 & 2.0 & 3.0 & 101 \\
0.1 & 8.625 & 8.630 & 8.636 & 8.55 \\
0.25 & 8.637 & 8.664 & 8.661 & 8.28 \\
0.50 & 8.682 & 8.750 & 8.684 & 8.77 \\
1.00 & 8.820 & $9.018^{2}$ & 8.860 & 8.32 \\
\hline
\end{tabular}

The constant $P$ for an insulated side wall; $x_{0}=2.0 \times 10^{5}$

1 Results for $(\lambda \gamma)>1.0$ are of questionable value.

2 Apparently picked up second eigenvalue.

Results for an insulated side wall are presented in Table 2 for $\lambda \leqslant 1.0$ and aspect ratio $\gamma=1,2,3$ and 10 . Several of these values, particularly the last column, are of questionable quantitative significance since they violate the restriction $\lambda \gamma \leqslant O(1)$ which must be satisfied in order to ensure reasonable accuracy for the basic state temperature profile represented by the polynomial expansion. For $\lambda \leqslant 3.0$, the results predicted for an insulated side wall do not differ greatly from those predicted for a conducting side wall. Although the results for a larger aspect ratio $(\gamma=10.0)$ are less accurate, they indicate that the basic state convection may have a destabilizing effect, that is the critical Rayleigh number is less than that which is predicted by an analysis of solid body rotation in a radially infinite container $(P=8.6959)$. This destabilization could occur through a distortion of the basic state temperature profile near the cylinder side wall. However, it should be noted that the difference between the calculated values of $P$ and the value obtained when basic state convection is neglected $(P=8.6959)$ is much less than the $30 \%$ observed by Rossby [20]. Thus even though the range of 
parameters treated in this paper does not include the conditions at which Rossby worked, it would seem that the lowering of the critical Rayleigh number observed by Rossby cannot be explained by a distortion of the basic state temperature profile via convection.

\section{§ 5. Disturbance velocity profiles}

The values of the expansion coefficients $w_{i, j}$ were determined to within $0.01 \%$ using the calculated $P$ and inverse iteration. The disturbance velocities at the point of inception are thus known from (29). The disturbance flow pattern is a very large number of axially symmetric ring cells, the widths of which are approximately the same as those found for instabilities in solid body rotation. The ring cells are superimposed on the basic state convection pattern. The characteristics of these ring cells can be described by considering only the vertical disturbance velocity $w$. The maximum value for each cell occurs on a horizontal plane midway between the top and bottom of the cylinder, i.e., $z=0.5$ and at the boundary between adjacent cells. These maxima are plotted verses radial position for a conducting side wall in Fig. 5 and an insulated side wall in Fig. 6. In both cases the parameter $\lambda=0.1$ and the aspect ratio $\gamma=1.0$. The scale on the ordinate is arbitrary since the problem is linear. The important feature is that the disturbance velocity at the point of instability is much greater near the outer edge of the cylindrical container than near the center. This should

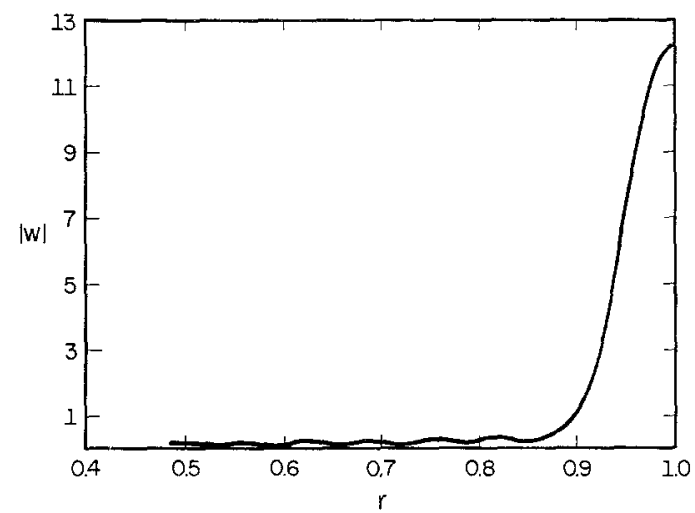

Fig. 5. Maximum axial velocity in a cell verses radial position. Conducting side wall. $\lambda=\sigma \beta \varepsilon^{-\frac{1}{2}}=0.1$; aspect ratio $\gamma=1.0$ 


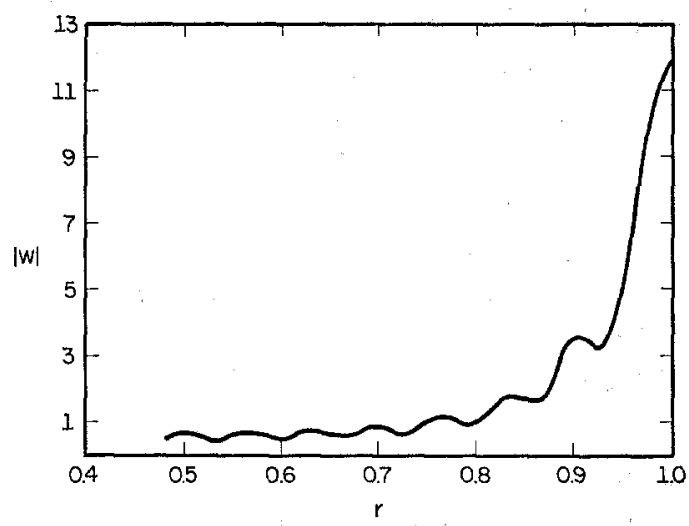

Fig. 6. Maximum axial velocity in a cell verses radial position. Insulated side wall. $\lambda=\sigma \beta \varepsilon^{-\frac{1}{2}}=0.1$; aspect ratio $\gamma=1.0$.

be contrasted with an analysis of stability of a fluid in solid body rotation where it is predicted that the magnitude of the disturbance velocity is the same in each cell. If these disturbance velocities are taken to be an adequate indication of what would be observed in a rotating cylinder at or slightly above the critical Rayleigh number, the instability should set in at the outer edge of the container. This seems to be in agreement with the observations of Koschmieder $[12,13,14]$.

\section{\$ 6. Conclusions}

When $\lambda<1.0$, the critical Rayleigh number differs little from that predicted assuming that the fluid is in solid body rotation. For $\lambda>1.0$, the fluid is stabilized by the basic state convection. The disturbance velocities are significantly influenced by the basic state convection in all cases. The magnitude of the disturbance velocity is much greater near the outer edge of the cylinder than near the center.

\section{Acknowledgment}

Grateful acknowledgment is made to the National Science Foundation for partial support of this work through Grant NSF GK 2505 and NSF GK 35760. 


\section{REFERENCES}

[1] Chandrasekhar, S., Proc Roy Soc A217 (1953) 306.

[2] Chandrasekhar, S. and D. D. Elaert, Proc Roy Soc A231 (1955) 198.

[3] Chandrasekhar, S., Hydrodynamic and Hydromagnetic Stability, The Clarendon Press, Oxford (1961).

[4] Finlayson, B., J. Fluid Mech. 33 (1968) 201.

[5] Veronis, G., J. Fluid Mech. 24 (1966) 545.

[6] Veronis, G., J. Fluid Mech. 31 (1968) 113.

[7] Niller, P. P. and F. E. Bisshopp, J. Fluid Mech. 22 (1965) 753.

[8] Homsy, G. M. and Hudson, J. L. J. Fluid Mech. 45 (1971) 353.

[9] Barcilon, V. and J. Pedlosky, J. Fluid Mech. 29 (1967) 673.

[10] Homsy, G. M. and J. L. Hudson, J. Fluid Mech. 35 (1969) 33.

[11] Homsy, G. M. and J. L. Hudson, J. Fluid Mech. 48 (1971) 605.

[12] Koschmieder, E. L., Beitr. Phys. Atmos. 39 (1966) 208.

[13] Koschmieder, E. L., Beitr. Phys. Atmos. 40 (1967) 216.

[14] Koschmieder, E. J., I. Fluid Mech. 33 (1968) 515.

[15] Luke, Y. L., Integrals of Bessel Functions, McGraw-Hill, New York (1962) 260.

[16] Watson, G. N., Treatise on the Theory of Bessel Functions, Cambridge, England (1958).

[17] Torrest, M. A., M. S. Thesis, University of Illinois, Urbana, Illinois (1970).

[18] Parlett, B., Math. Comp. 18 (1968) 464.

[19] Wilkinson, J. H., The Algebraic Eigenvalue Problem, The Clarendon Press, Oxford (1965).

[20] Rossby, H. T., J. Fluid Mech. 36 (1969) 309. 